\title{
HIGH-DISPERSION SPECTROSCOPY OF IC 351 AND NGC 3242, PLANETARIES WITH HIGH INTERNAL MOTION
}

\author{
Y.YADOUMARU and S.TAMURA \\ Astronomical Institute, Tohoku University, Aoba-ku Sendai 980, Japan
}

We made high-dispersion spectroscopic observations of IC $351=$ PK $159-15^{\circ} 1$, a compact high-excitation planetary nebula. We found the expansion velocity from $\mathrm{H} \alpha,[\mathrm{OIII}]$ and $\mathrm{HeII}$ line, of $45.1 \mathrm{kms}^{-1}, 38.7 \mathrm{kms}^{-1}$, and $33.3 \mathrm{kms}^{-1}$, respectively. Moreover, for the $\mathrm{H} \alpha$ line we detected an additional faint blue-shifted component escaping from the center of expansion with high velocity of $120 \mathrm{~km} \mathrm{~s}^{-1}$. This value is larger than the usual expansion velocity $\left(<50 \mathrm{kms}^{-1}\right)$ but considerably smaller than that of stellar wind. This faint blue-shifted component of $\mathrm{H} \alpha$ is also coincidently identified as the helium line, HeII $\lambda 6560$ (Pickering 6). Using the emission coefficients of recombination lines (Case $B, T=10,000 \mathrm{~K}$; Osterbrock 1989), and the intensity ratio, $\mathrm{I}(\mathrm{HeII} \lambda 4686) / \mathrm{I}(\mathrm{H} \beta)=0.56 \pm 0.05$ (Aller\&Czyzak 1979), we can estimate the expected line intensity ratio, $\mathrm{I}(\mathrm{HeII} \lambda 6560) / \mathrm{I}(\mathrm{H} \alpha)=0.026 \pm 0.002$. On the other hand, the intensity ratio of faint component to $\mathrm{H} \alpha$ in the present observations is estimated as $\mathrm{I}_{\text {faintcomp. }} / \mathrm{I}(\mathrm{H} \alpha)=0.064 \pm 0.013$, based up the $\mathrm{H} \alpha$ profile in 1988. Comparing these two estimated intensity ratios it is clearly unable to emit the all intensity of the observed faint component by HeII $\lambda 6560$ line only. Therefore, even if this component is partly polluted by HeII $\lambda 6560$ line, we can consider the rest part as an emission from high velocity component of $\mathrm{H} \alpha$. We can also recognize the bumped feature of [OIII] $\lambda 5007$ line around the same velocity to the blue-shifted component of $\mathrm{H} \alpha$. This fact supports the idea that these components can be attributed to the high velocity flow. We discuss the three ideas for the interpretation of such component; (a) colliding winds, (b) unresolved bipolar flow, and (c) secondary formation of an expanding shell. We have investigated whether the colliding winds model gives such high velocity or not. As the results of the calculation, if we use the usual mass-loss rate, the model can not give such high velocity. Therefore, it is doubtful that we can take this model as an explanation of the high velocity components. The high velocity component of IC 351 is not uncommon; such large internal motions are reported for other planetaries by Weinberger(1989) and compiled by Grewing(1989). The article on the results of IC 351 is submitted to PASP (Yadoumaru \& Tamura 1992). We also report the other analysis on NGC 3242.

\section{References}

Aller L.H. and Czyzak S.J.,1979,Ap\&SS,62,397.

Grewing M.,1989,in "IAU Symposium No.131, Planetary Nebulae", ed. Torres-Peimbert S.,(Reidel, Dordrecht, Holland),p.241.

Osterbrock D.E.,1989, Astrophysics of Gaseous Nebulae and Active Galactic Nuclei,p.80 and p.85.

Weinberger R.,1989,in "IAU Symposium No.131, Planetary Nebulae", ed. Torres-Peimbert S.,(Reidel, Dordrecht, Holland),p.93.

Yadoumaru Y. and Tamura S.,1992,submitted to PASP. 